\title{
Numerical Errors - Numerical Uncertainty - in the Identification and Estimation of Vibroacoustic Processes
}

\author{
W. BATKO \\ Department of Mechanics and Vibroacoustic, AGH, University of Science and Technology \\ al. Mickiewicza 30, 30-059 Kraków, Poland
}

\begin{abstract}
Problems of numerical errors in the identification and estimation procedures of vibroacoustic phenomena are discussed in the hereby paper. The main attention is focussed on problems of errors generated by different algebras governing experimentally recognisable vibroacoustic effects and the algebra applied in the utilisation of procedures of their processing. Assessment of errors for elementary operations: adding, subtracting, multiplying and raising to a power - is derived. The problem of their presence in the current model solutions as well as in the implications for processes: cognitive, experimental (testing conditions) and their implementation is discussed. The postulate of the necessity of supplementing assessments of the measurement uncertainty in vibroacoustic examinations with assessments of the numerical uncertainty - are formulated. Various ways of looking for solving this problem are outlined. References to Author's and co-authors' own investigations — in this field — are also done.
\end{abstract}

PACS numbers: 43.40.At

\section{Introductory comments}

Vibroacoustics being a science which deals with all vibratory and acoustic processes - occurring in the nature and technique - as well as with their harmful influences on life and work environments — is par excellence an empirical science.

This means that the majority of identification requirements, i.e. relations (models) describing the determined acoustic and vibratory influences are related to their empirical experiences. The human reception mechanism transferred into the controlling operation of the measuring equipment is essential in their experimental recognition.

It is stated by the Weber-Fechner rule, that the human reception of vibroacoustic stimuli is proportional to the logarithm of the quotient of these process changes with respect to the references determined for them. Thus, the measuring parameters appearing in the description of the identified vibroacoustic models of the analysed effects are values of acoustic pressure levels, vibration levels or other units of the stimulus influencing the human organism. This stimulus is expressed in units of the given influence level, described by decibel measures [dB].

In case of the recognition of the mechanism of acoustic effects they are being described by changes of the acoustic pressure level: $L=10 \log \left\{p^{2} / p_{0}^{2}\right\}[\mathrm{dB}]$.

Their values are determined by 10 decimal logarithms, from the ratio of the acoustic pressure square $p(t)$ and the reference pressure $p_{0}=2 \times 10^{-5} \mathrm{~Pa}$, which is a threshold of hearing of sounds of a frequency equal $1000 \mathrm{~Hz}$.

That is the reason that data collections - obtained in experimental cognitive processes of various vibroacous- tic effects $-L_{i} ; i=1,2, \ldots, n$ are expressed in decibel units. They constitute the identification basis for the construction of vibroacoustic models. Using of such data collections in the identification process generates certain problems related to the algebra governing their processing, resulting from the law of their mutual co-operation $[1,2]$.

The presented work aims to provoke the discussion concerning the basic problem of several vibroacoustic investigations, which is the question: whether an application of the universal - available in the market - software for the measurement data exploration (data mining) and for the identification of parametric models is fully justified. Especially not critical use of the algorithms and assumptions determining their software realisation — not having a reference to the modelled vibroacoustic effects identified by measurements expressed in decibel units - is rather doubtful. Purposefulness of the discussion about the vibroacoustic models quality - in respect of their cognitive values - is related to the lack of uncertainty assessments of the model solutions obtained this way.

There is a lack of uncertainty assessments generated by numerical errors related to the conversion of the results data bases of the sound level measurements according to the classic algebra rules, without taking into account different interrelations $[4,6]$ of the investigated sound levels, from various sources, in such elementary operations as: adding, subtracting or multiplying.

Deliberation on this problem is the aim of the present paper. The attention will be directed towards the analysis of errors, which can accompany this process. Possible consequences of not taking into account those effects in 
uncertainty assessments for: cognitive and experimental processes (conditions of performing examinations) as well as their applications are outlined. The postulate of the necessity of supplementing the hitherto realised assessments of measuring uncertainty in vibroacoustic investigations by assessments of numerical uncertainty is formulated. The presented discussion is aimed at the development of logical bases for vibroacoustic modelling, since it can be useful not only for vibroacoustics but also for other fields of empirical sciences, which use the data measured in decibel units.

\section{Analysis of model and identification conditions in vibroacoustic investigations}

Acoustic and vibration theories (well known in general, providing the quantitative description of interesting effects: their generation and propagation) are used in vibroacoustic investigations. It would be difficult to point out any significant achievements of vibroacoustics, which would not be referred to the fundamental physical knowledge and theory language: acoustics, vibrations, equations presenting the quantitative description of the analysed effect. However, empirical experiments data are quite often needed for the explanation of vibroacoustic effects. They are found by measuring sound levels - determining those effects - expressed in decibel units, as well as from numerous data sets storing information on those processes. If those sets are sufficiently numerous they can allow for the identification of the model simulating original effects occurring in the analysed vibroacoustic effects. The model obtained in such way can be useful in various applications. We are dealing with such situation in forecasting the sound level distribution - generated by various sources - in places subjected to the environmental protection. This identification technique is more and more often used in numerous researches of the vibroacoustic field. It is related — on the one hand to the development of new and more advanced computer tools for collecting, storing and processing of large data bases and - on the other hand - to more numerous vibroacoustic problems requiring the identification of the governing rules.

The purpose of the presented paper is to point out, to potential researchers - using this way of recognition in vibroacoustics - the related limitations and possible errors at endeavours of the vibroacoustic models identification.

Rules of algebraic calculations of experimentally found acoustic effects are different than rules applied for their processing and the algebra present in numerous software tools used for the identification and compilation of experimental results.

The following relations govern the experimentally recognisable acoustic effects, in which the result is given by the logarithmic measure: "addition", "subtraction" and "multiplication". For the co-operation of two acoustic events determined by the measured sound levels:
$L_{1}=A \mathrm{~dB}$ and $L_{2}=B \mathrm{~dB}$ the following equations are given:

$$
\begin{aligned}
& \text { Addition "+" } \\
& A+B=10 \log \left(10^{0.1 A}+10^{0.1 B}\right)
\end{aligned}
$$

Subtraction "-"

$$
A-B=\log \left(10^{0.1 A}-10^{0.1 B}\right)
$$

Multiplication " $x$ "

$$
k \times A=10 \log \sum_{i=1}^{k} 10^{0.1 A}=A+10 \log k
$$

When these rules are disregarded in identification algorithms of the vibroacoustic model sought after, they will generate - in the reactions attributed to them the determined errors of differences:

Error of the addition operation $\delta_{+}$

$$
\begin{aligned}
& \delta_{+}=A+B-10 \log \left(10^{0.1 A}+10^{0.1 B}\right) \\
& =A+B-10 \log 10^{0.1 A}\left(1+10^{0.1(B-A)}\right) \\
& =A+B-A-10 \log \left(1+10^{-0.1(A-B)}\right) \\
& \delta_{+}=B-10 \log \left(1+10^{-0.1 \Delta}\right) \quad[\mathrm{dB}] \\
& \text { where: } \Delta=A-B \\
& \text { Error of the subtraction operation } \delta_{-} \\
& \delta_{-}=A-B-10 \log \left(10^{0.1 A}-10^{0.1 B}\right) \\
& =A-B-10 \log 10^{0.1 A}\left(1-10^{0.1(B-A)}\right) \\
& =A-B-A-10 \log \left(1-10^{-0.1(A-B)}\right) \\
& \delta_{-}=-B-10 \log \left(1-10^{-0.1 \Delta}\right) \quad[\mathrm{dB}] \\
& \text { where: } \Delta=A-B
\end{aligned}
$$

Error of the multiplication operation $\delta_{\times}$

$$
\begin{aligned}
\delta_{\times} & =k \times A-A-10 \log k \\
& =(k-1) \times A-10 \log k \quad[\mathrm{~dB}]
\end{aligned}
$$

The essential limitation, in using standard identification procedures in reference to the basis of the measuring results and to the excitations determining their changes, is the inability of accepting assumptions, at which they were developed and programmed.

Algorithms assigned to numerous software packages (dedicated to identification procedures) were derived at the assumption that the set of the analysed results is determined in the metric space with the determined metric. This metric, usually Euclidean, is a square root of the sum of squares of distances of the analysed results. It means, that to each pair of arbitrary elements $x, y$ from the set $\boldsymbol{X}$ of the measurement results the distance metric $\rho(x, y)$ can be assigned, fulfilling — for its arbitrary elements $x, y, z \in \boldsymbol{X}$ the following conditions:

$$
\begin{aligned}
& \rho(x, y) \geq 0 \text { and } \rho(x, y) 0 \\
& \text { when and only when } x=y \\
& \rho(x, y)=\rho(y, x) \\
& \rho(x, y) \leq \rho(x, z)+\rho(z, y)
\end{aligned}
$$

It can be easily noticed that the set of measurement re- 
sults determined by sound level values and conditions of their addition (1) does not meet the condition of triangle in the Euclidean metric (9) (e.g. for the results: $40 \mathrm{~dB}$, $44 \mathrm{~dB}, 50 \mathrm{~dB}$ the sum $40 \mathrm{~dB}+44 \mathrm{~dB}$ is not larger than $50 \mathrm{~dB}$ ). If this fact is not taken into account when using the universal identification software (present in numerous packets) it can become a source of an unsatisfactory recognition of the vibroacoustic effects under testing.

Thus, asking for the level of errors related to these conditions seems to be justified. This question should become the stimulus for undertaking broader investigations and the basis for an important discussion on limitations related to the application of classic algorithms in reference to the sound level results. This means the need of introduction the component assessing their numerical uncertainties - into the principles of the uncertainty assessment in the identification and estimation procedures of vibroacoustic effects. This component can be generated by the lack of the possibility of accepting assumptions applied for working out the experimental results $[3-5]$.

The grounds for such discussion should constitute an agreement on the criteria according to which certain procedures could be either accepted or rejected. Their basis should be the properly defined (for the realised identification conversions of acoustic measurement results) metric space with a metric fulfilling the condition (9) and the operators of adding " + ", subtracting "-_, or multiplying " $x$ " defined by conditions adequate for the co-operation of acoustic sound levels (1-3).

\section{Numerical uncertainty of acoustic models obtained on the basis of exploration of the measurement data (data mining)}

As it was mentioned in the previous paragraph, the essential space of the acoustic effects recognition constitute experimental investigations. In those experiments the acoustic relations describing the investigated reality fragment are obtained, by means of data mining from multidimensional sets of the acoustic measurement results - determined by decibel measures (aided by the software for their processing). Thus, it is understandable, that when the detailed knowledge on the nature of the relations under testing is not available, such model is just one out of several possible models selected by assuming a certain criterion of the model error. Thereby the selection of the criterion is essential in the development of the identification procedures. This criterion should enable such selection (from numerous possible admissible descriptions of the tested relations) of the model, that it would have the highest probability of being a good model not only for the investigated case, but also for other applications. The correctly developed model of acoustic effects is a valuable inference tool not only for the analysed measurement data but also for other similar cases. Respecting the Heisenberg Uncertainty Principle, which states that the future of every analysed effect retains a certain degree of uncertainty and randomness, one is not allowed to disregard the question: to what degree the approximating function should be smoothing changes of the analysed effects and whether disregarding of extreme behaviours - applied in a classic model building - is justified in case of vibroacoustic investigations.

This problem - in relation to the analysed acoustic events - is connected with selecting the metric for their comparisons. The sought after relations - describing the acoustic effects under testing — are being derived by means of this metric. The Euclidean metric, being the basis of numerous identification algorithms of models and their implementations, is not suitable for comparisons analysed in the acoustic effects investigations. It does not satisfy the triangle inequality condition in respect of comparisons of the sound level results expressed by a decibel measure (9). Dominating influences are specially important in the identification investigation process, and this is expressed by relations (1) and (2). It is worth consideration, why those conditions have not been reflected in hitherto assessments of the realised investigations. Analysis of errors generated by not correctly performed operations of mutual co-operations of the analysed acoustic events (described by decibel measures) can partially explain the situation. According to relations (5-6) presented in the previous paragraph, the error related to these operations is significantly lower than the dominating level. Thus, it is difficult to notice it during the measuring process. However, it does not mean that it should not be discussed.

Omitting it - in the identification process of principles governing the investigated effects - constitutes the source of the determined errors. They can have an essential influence on uncertainty assessments of the obtained solutions. Principles of assessments of measurement uncertainties $[6,7]$ impose the necessity of:

- Assessment of the measurement uncertainty for each investigated process included in the measurements management system;

- Assessment of uncertainty of all possible discriminants of the investigation process and related to it sources generating its variability;

- Assessment of errors of all possible discriminants, which are essential in the control process, with the application of the proper methods of analysis.

They also permit the possibility of the resignation from assessing some uncertainty components, when they are negligible as compared to other ones and generate unreasonable technical and economical expenditures. In accordance with these principles, there is a need of a broader determination of numerical errors influences, generated by the described above conditions, on the realised assessment results of the investigated acoustic effects.

Analysing consequences, of the described above uncertainties of numerical calculations, for the recognition 
process of vibroacoustic effects, the fundamental question should be asked: "Where is the efficiency limit of the worked out model solutions?". It can be judged, that a certain limited recognition of the identified relations - not allowing to notice some details - is related to the discussed numerical errors. A good model, free from those limitations, it means derived on the properly selected metrical space versus the set of acoustic measurement results determined by sound levels $[\mathrm{dB}]-$ with correctly defined algebraic operations assigned to co-operation conditions of sound sources - can refine experiences. It can be expected, that conditions for the recognition of facts, which were unnoticeable by models identified by hitherto algorithms, would occur. The knowledge of the uncertainty of numerical calculations can be also useful in selecting the grade of instruments used in measurements.

The need of assessments of numerical errors in experimental acoustic investigations can be also connected to the administrative utilisation of the sound level results as happens in environmental control results. Administrative decisions depend on these results. They can concern the imposed penalties in cases of the excessive emission of sound levels into the environment by the determined subject as well as the requirement of introduction certain measures to limit noises in the environment. Uncertainty assessments related to these conditions can be important factors useful in taking administrative decisions concerning the acoustic environment management [8,9].

\section{Final remarks}

Summarising, it should be stated, that there is a necessity of analysing uncertainties of numerical calculations in numerous investigations of acoustic effects carried out by the exploration of large sets of the sound level results. This need is caused by the numerical errors generated by the fact, that one type of algebra is governing the acoustic effects and the different one is used in calculations of the experimental results - implemented in the identification and estimating algorithms being commonly used. The source of errors constitutes also the metric of comparing the analysed sound levels (not correctly assumed in their realisation). As can be seen, on the basis of the presented considerations and analyses of the possible errors related to operations of adding, subtracting and multiplying sound levels, there is the need of defining the algebra for processing the measurement results (with the selection of the distance metric suitable for their comparisons).

The attention was also focused on consequences corresponding to the presence of uncertainty in numerical calculations in the investigation process. It was discussed from the point of view of the realised cognitive and experimental (conditions of performing acoustic investigations) processes as well as related to them economic and legal conditions.

The potential mathematical formalism for solving the discussed problem was outlined. The need of undertaking broader research concerning the numerical uncertainty in vibroacoustic investigations - being the condition for further development - was substantiated.

If the proper methodological frame for the exploration of large sets of the sound level measurement results together with the basis of identification methods assigned to them is built, the further progress of vibroacoustics will be expected.

\section{References}

[1] H.J. Bress, Rechnen mit Pegelgroessen, Mathematischer Umgang mit Dezibelwerten, K. Larsen, Glostrup 1987, 2 Auflage.

[2] H. Hoffman, A. Luepke, 0 Dezibel +0 Dezibel $=3$ Dezibel, Erich Schmidt Verlag, Berlin 1975.

[3] W. Batko, R. Bal, Scientific reports - University of Applied Sciences Mittweida 9, 15 (2008) (in German).

[4] W. Batko, R. Bal-Pyrcz, Arch. Acoustics 31, 4 (supplement), 253 (2006).

[5] W. Batko, B. Stępien, Archives of Acoustics, submitted for print.

[6] International Organization for Standardization, Guide to the expression of uncertainty in measurements, (1995).

[7] PN-EN ISO 10012 - 2004, Systems of Measurement Management. Requirements Concerning Measuring Processes and Equipment.

[8] Directive 2002/49/WE of the European Parliament and of the Council of 25 June 2002, related to the assessment and management of environmental noise, Official Journal of the European Communities 18.07.2002.

[9] J. Law, 62, 627 (2001). 